\title{
A No-Equilibrium Hyperchaotic System and Its Fractional-Order Form
}

\author{
Duy Vo Hoang, ${ }^{1}$ Sifeu Takougang Kingni, ${ }^{2}$ and Viet-Thanh Pham ${ }^{3}$ \\ ${ }^{1}$ Modeling Evolutionary Algorithms Simulation and Artificial Intelligence, Faculty of Electrical \& Electronics Engineering, \\ Ton Duc Thang University, Ho Chi Minh City, Vietnam \\ ${ }^{2}$ Department of Mechanical and Electrical Engineering, Institute of Mines and Petroleum Industries, University of Maroua, \\ P.O. Box 46, Maroua, Cameroon \\ ${ }^{3}$ School of Electronics and Telecommunications, Hanoi University of Science and Technology, 01 Dai Co Viet, \\ Hanoi, Vietnam \\ Correspondence should be addressed to Viet-Thanh Pham; pvt3010@gmail.com
}

Received 25 March 2017; Accepted 1 June 2017; Published 29 June 2017

Academic Editor: Jonathan N. Blakely

Copyright (C) 2017 Duy Vo Hoang et al. This is an open access article distributed under the Creative Commons Attribution License, which permits unrestricted use, distribution, and reproduction in any medium, provided the original work is properly cited.

No-equilibrium system with chaotic behavior has attracted considerable attention recently because of its hidden attractor. We study a new four-dimensional system without equilibrium in this work. The new no-equilibrium system exhibits hyperchaos and coexisting attractors. Amplitude control feature of the system is also discovered. The commensurate fractional-order version of the proposed system is studied using numerical simulations. By tuning the commensurate fractional-order, the proposed system displays a wide variety of dynamical behaviors ranging from coexistence of quasiperiodic and chaotic attractors and bistable chaotic attractors to point attractor via transient chaos.

\section{Introduction}

It is now well established from a variety of studies that a hyperchaotic system is specified by having at least two positive Lyapunov exponents [1]. Hyperchaotic systems have been applied in various areas due to their higher level of complexity with respect to chaotic systems [2-6]. There has been an increasing amount of literature on hyperchaos $[7,8]$. Authors have introduced and studied different hyperchaotic systems such as switched hyperchaotic system [9], four-wing hyperchaotic attractor [10], hyperchaotic Chua's circuits [11], and hyperchaotic Lü attractor [12]. It is noted that there are countable numbers of equilibrium points in such reported hyperchaotic systems.

It is interesting that Wang et al. found a hyperchaotic system without equilibrium [13], which is different from normal hyperchaotic systems. Following up the first discovery of Wang et al., other hyperchaotic systems without equilibrium were presented [14-17]. Recent studies have attempted to explore special features of no-equilibrium hyperchaotic systems. Wang et al. investigated multiwing nonequilibrium attractors in simplified hyperchaotic systems [18]. Bao et al. constructed a memristive hyperchaotic system which does not display any equilibrium [19]. This memristive system generated coexisting hidden attractors. Moreover, fractionalorder systems without equilibria were discovered in [20,21]. It is worth noting that attractors in such no-equilibrium hyperchaotic system are "hidden" from the viewpoint of computations $[22,23]$. More recent attention has focused on hidden attractors due to their importance in both theoretical issues and engineering applications [24-27].

The aim of this study is to introduce a new hyperchaotic system without equilibrium. The organization of the paper is as follows. The new no-equilibrium system is described in the next section. Section 3 presents dynamics of the new system without equilibrium. Fractional derivation effect on the proposed system is investigated in Section 4. Our conclusions are drawn in the last section. 


\section{The Model of the New System without Equilibrium}

Wei and Wang have introduced a special system which is different from the original Lorenz and Lorenz-like systems [28]. Wei-Wang system is given by

$$
\begin{aligned}
& \dot{x}=a(y-x), \\
& \dot{y}=-b y+n x z, \\
& \dot{z}=d-e^{x y},
\end{aligned}
$$

in which $x, y$, and $z$ are state variables while $a, b, d$, and $n$ are parameters. There are two equilibrium points in system (1) for $d>0$ and $n \neq 0: E_{1}(\sqrt{\ln d}, \sqrt{\ln d}, b / n)$ and $E_{2}(-\sqrt{\ln d}$, $-\sqrt{\ln d}, b / n)$. Moreover, Wei-Wang system (1) is chaotic when both equilibrium points $E_{1,2}$ are stable [28].

Motivated by the noticeable features of model (1), we construct a novel four-dimensional system by introducing an additional fourth state $w$ as follows:

$$
\begin{aligned}
\dot{x} & =a(y-x), \\
\dot{y} & =-b y+n x z+c w, \\
\dot{z} & =d-e^{x y}, \\
\dot{w} & =-m y .
\end{aligned}
$$

in which $x, y, z$, and $w$ are four state variables. In system (2), positive parameters are $a, b, c, d, n, m$, and $d \neq 1$. System (2) is invariant under the transformation $(x ; y ; z ; w) \rightarrow$ $(-x ;-y ; z ;-w)$. It means that the system has rotational symmetry around the $z$-axis.

Obviously, it is simple to find equilibrium points of system (2) by solving $\dot{x}=0, \dot{y}=0, \dot{z}=0$, and $\dot{w}=0$. Thus we get

$$
\begin{aligned}
a(y-x) & =0, \\
-b y+n x z+c w & =0, \\
d-e^{x y} & =0, \\
-m y & =0 .
\end{aligned}
$$

Therefore, there is not any equilibrium in system (2).

It is easy to see that no-equilibrium system (2) is dissipative with an exponential contraction rate

$$
\frac{d V}{d t}=e^{-(-a-b) t}
$$

because of

$$
\nabla V=\frac{\partial \dot{x}}{\partial x}+\frac{\partial \dot{y}}{\partial y}+\frac{\partial \dot{z}}{\partial z}+\frac{\partial \dot{w}}{\partial w}=-a-b<0 .
$$

However, it is noted that the dissipativity in the sense of Levinson should be studied further [29, 30].

It is interesting that the system without equilibrium exhibits hyperchaotic behavior for $a=n=1, b=$ $m=0.5, c=0.2$, and $d=2.5$ and initial conditions $(x(0), y(0), z(0), w(0))=(0.1,0.1,0.1,0.1)$ as illustrated in Figure 1. The Lyapunov exponents of the no-equilibrium system are $L_{1}=0.1515, L_{2}=0.0112, L_{3}=0$, and $L_{4}=-1.6623$. We have applied Wolf's method [31] to calculate the Lyapunov exponents. The time of computation is 10,000 and the initial conditions are $(x(0), y(0), z(0), w(0))=$ $(0.1,0.1,0.1,0.1)$. It is noted that the values of Lyapunov exponents are not the same for any initial point on invariant set [32]. Computations of Lyapunov exponents should be considered seriously [33-35].

\section{Dynamical Behavior of the System without Equilibrium}

Dynamics of the system has been investigated by considering the effect of parameters on system's behavior. Our simulations show that no-equilibrium system (2) displays rich dynamics for the parameter $a$. We have studied the dynamics of noequilibrium system (2) by varying the value of parameter a. Bifurcation diagram and Lyapunov exponents of noequilibrium system (2) are presented in Figures 2 and 3, respectively. For $a>2.276$, the system without equilibrium only generates periodical behavior. However, no-equilibrium system (2) displays different attractors such as periodic, quasiperiodic, chaotic, and hyperchaotic attractors for $a<$ 2.276 .

We have also investigated the multistability of the new no-equilibrium system by using the continuation diagram [36]. As can be seen in Figure 4, a window of multistability dynamics is identified in the range of $a$ from 1.905 to 2.215. For example, Figure 5 illustrates the coexisting attractor in no-equilibrium system (2) for $a=2.1$.

From the viewpoint of applications, the amplitude control of a chaotic signal is an important topic [37-40]. Interestingly, no-equilibrium system (2) is an amplitude-controllable system. Here we illustrate this special feature of no-equilibrium system (2). (2):

We introduce a single control parameter $k_{w}$ into system

$$
\begin{aligned}
& \dot{x}=a(y-x), \\
& \dot{y}=-b y+n x z+c w-k_{w}, \\
& \dot{z}=d-e^{x y}, \\
& \dot{w}=-m y .
\end{aligned}
$$

The control parameter $k_{w}$ is used to boost the amplitude of the variable $w$. Therefore, we can change the signal $w$ easily, for example, from a bipolar signal to unipolar one as illustrated in Figure 6.

In order to control the amplitudes of variables $x$ and $y$, the coefficient $k_{x y}$ is included into system (2) as follows:

$$
\begin{aligned}
& \dot{x}=a(y-x), \\
& \dot{y}=-b y+n x z+c w, \\
& \dot{z}=d-e^{k_{x y} x y}, \\
& \dot{w}=-m y .
\end{aligned}
$$




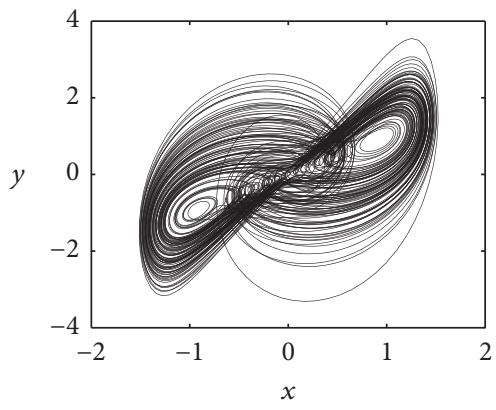

(a)

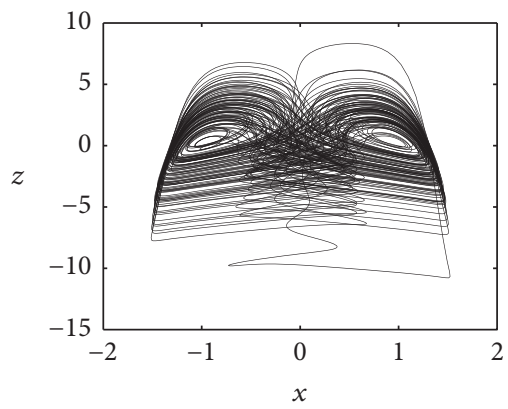

(b)

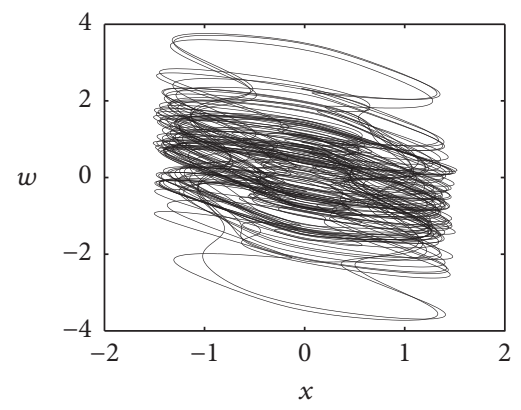

(c)

Figure 1: Projections of hyperchaotic attractors in (a) $x$ - $y$ plane, (b) $x$ - $z$ plane, and (c) $x$ - $w$ plane for $a=n=1, b=m=0.5, c=0.2, d=2.5$, and initial conditions $(x(0), y(0), z(0), w(0))=(0.1,0.1,0.1,0.1)$.

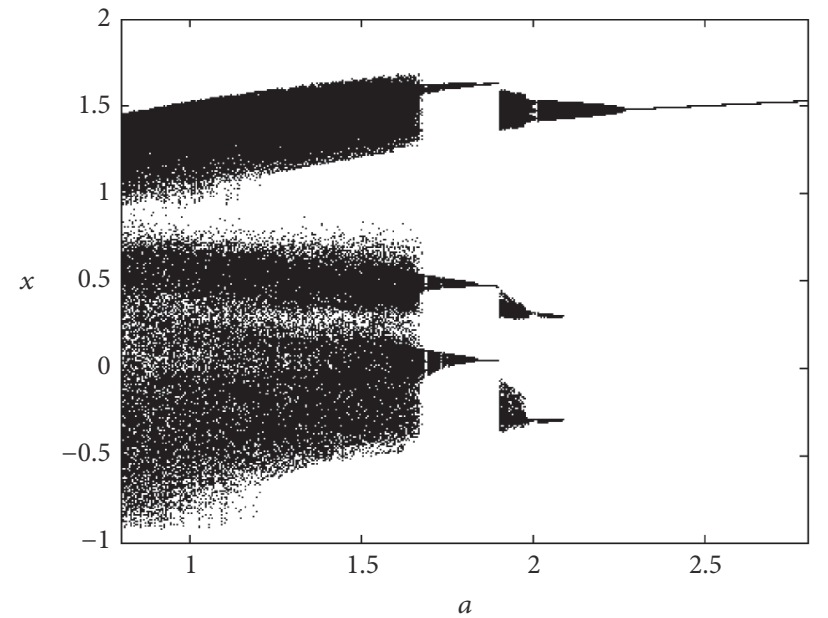

FIGURE 2: Bifurcation diagram of no-equilibrium system (2) for $b=m=0.5, c=0.2, d=2.5, n=1$, and $a \in[0.8,2.8]$.

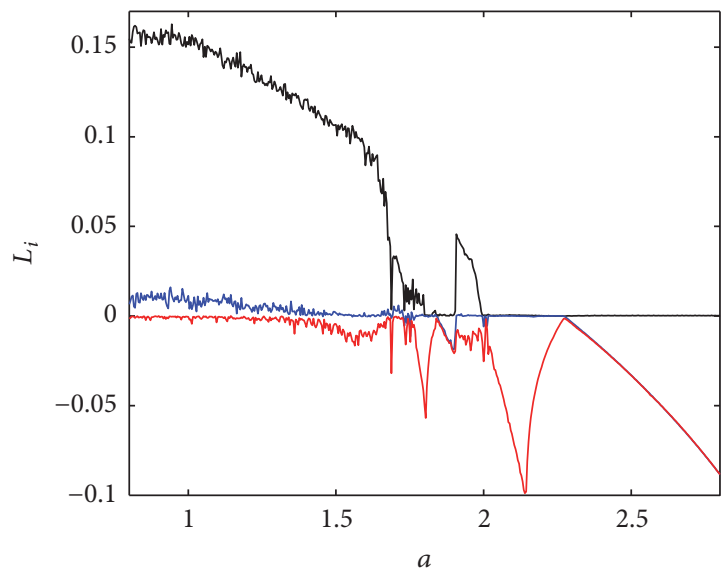

$$
\begin{aligned}
& -L_{1} \\
& -L_{2} \\
& -L_{3}
\end{aligned}
$$

FIGURE 3: Three largest Lyapunov exponents of no-equilibrium system (2) for $b=m=0.5, c=0.2, d=2.5, n=1$, and $a \in[0.8,2.8]$. 


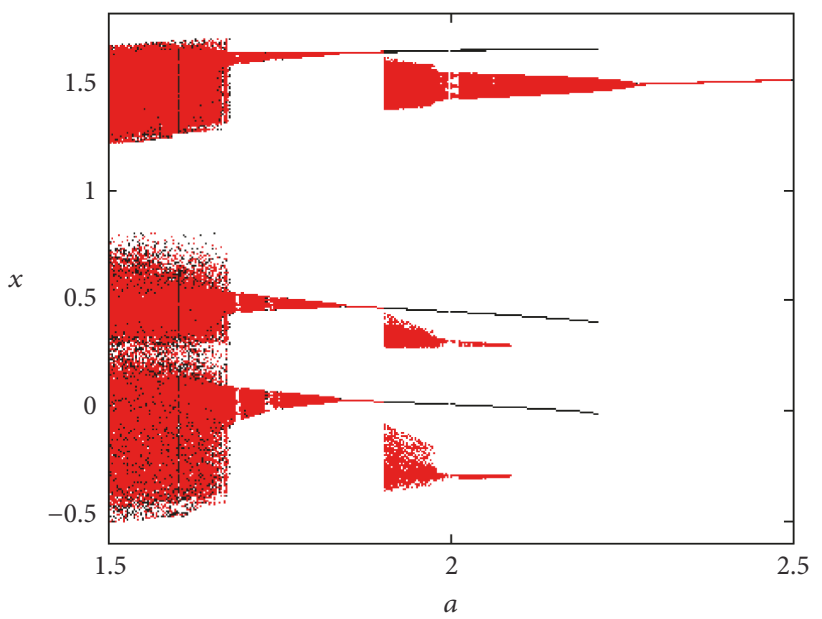

Figure 4: Continuations of new no-equilibrium system (2) for $b=m=0.5, c=0.2, d=2.5$, and $n=1$. Forward continuation (black): increasing the bifurcation parameter $a$ from 1.5 to 2.5 and starting with initial conditions $(x(0), y(0), z(0), w(0))=(0.1,0.1,0.1,0.1)$. Backward continuation (red): decreasing the bifurcation parameter $a$ from 2.5 to 1.5 and starting with initial conditions $(x(0), y(0), z(0), w(0))=$ $(-0.1,-0.1,0.1,-0.1)$.

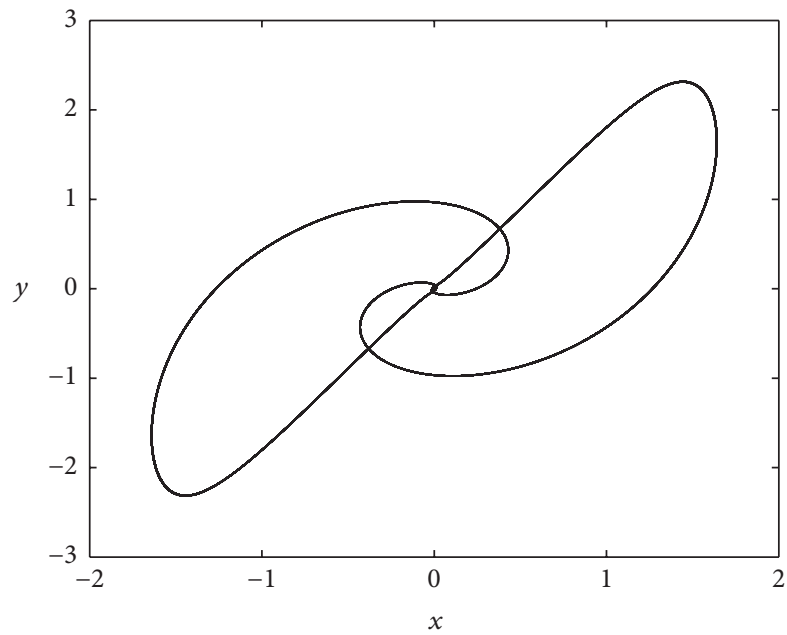

(a)

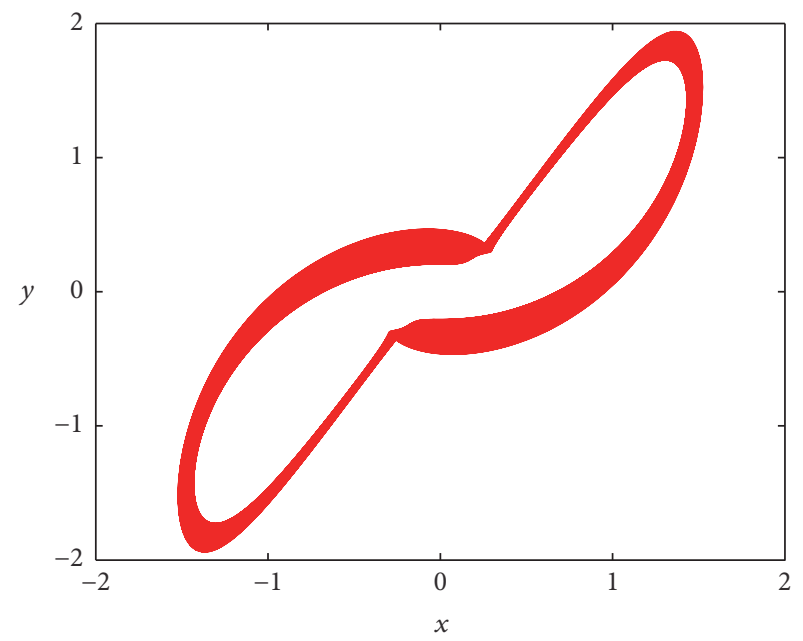

(b)

Figure 5: Coexistence of attractors in no-equilibrium system (2) for $a=2.1, b=m=0.5, c=0.2, d=2.5$, and $n=1$ in $x$ - $y$ plane: (a) periodic state and (b) quasiperiodic state.

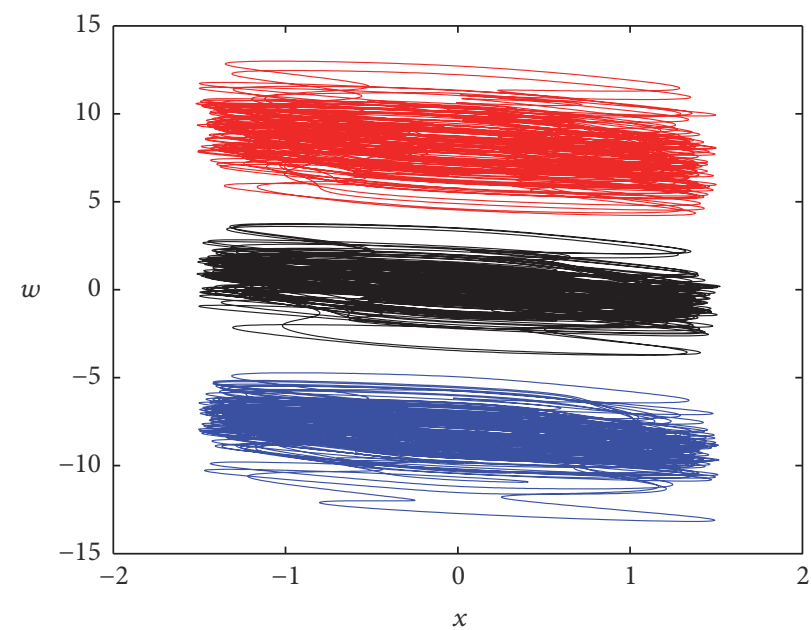

FIGURE 6: Varying attractors of no-equilibrium system (6) for $a=n=1, b=m=0.5, c=0.2$, and $d=2.5$ for different boosting constants: $k_{w}=0$ (black), $k_{w}=-1.6$ (blue), and $k_{w}=1.6$ (red). 


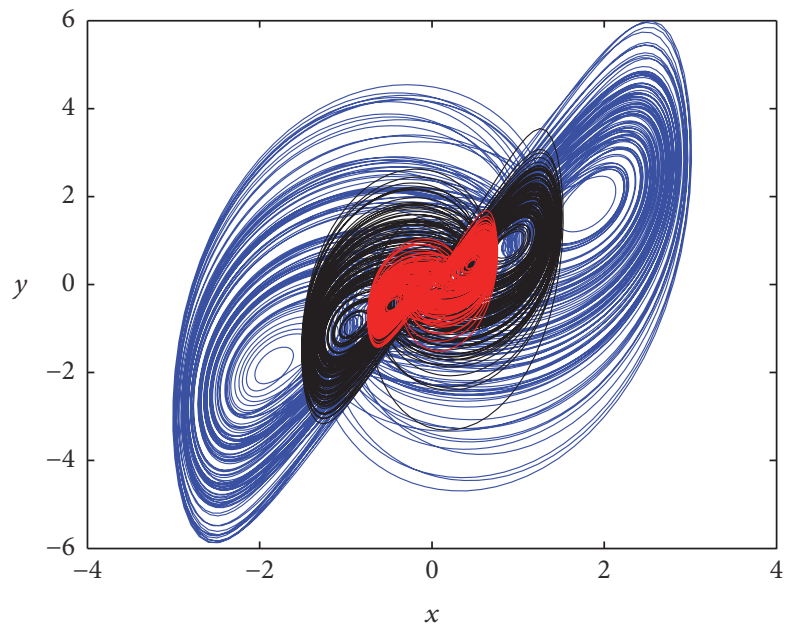

FiguRE 7: Controllable attractors of no-equilibrium system (7) for $a=n=1, b=m=0.5, c=0.2$, and $d=2.5$ when changing the coefficient $k_{x y}: k_{x y}=1$ (black), $k_{x y}=0.25$ (blue), and $k_{x y}=4$ (red).
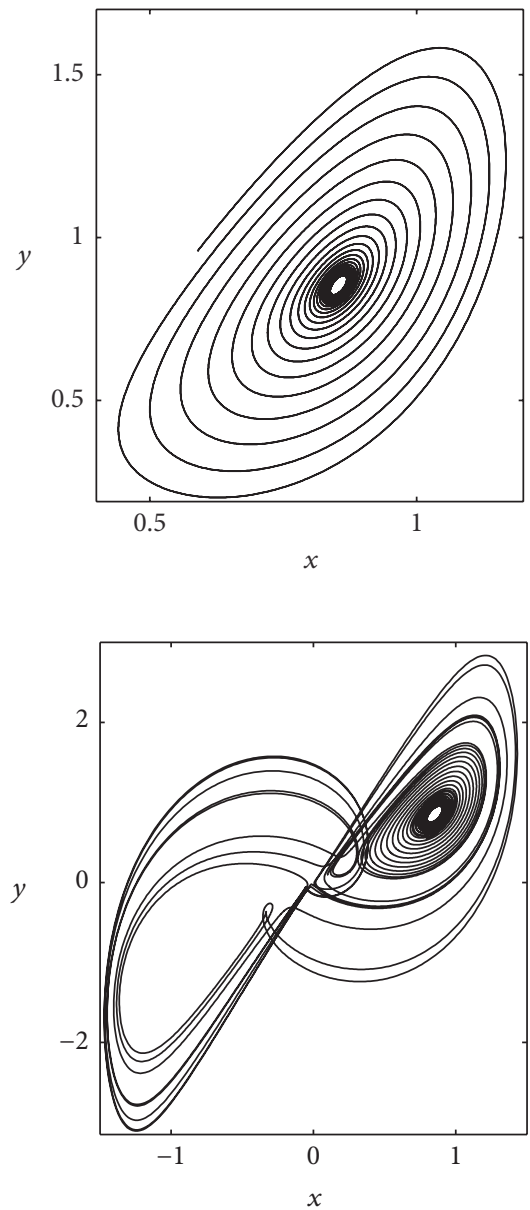

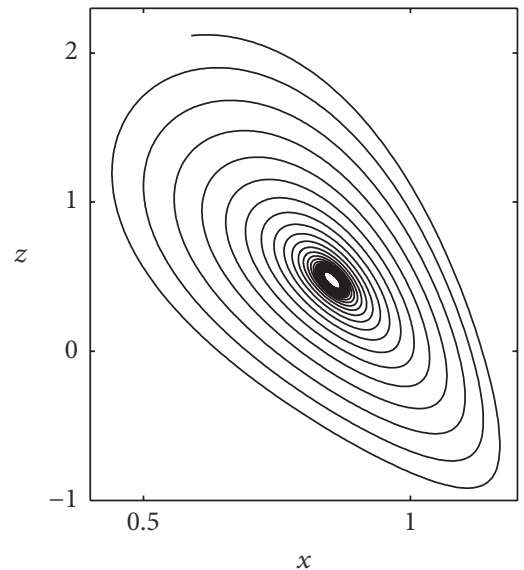

(a)

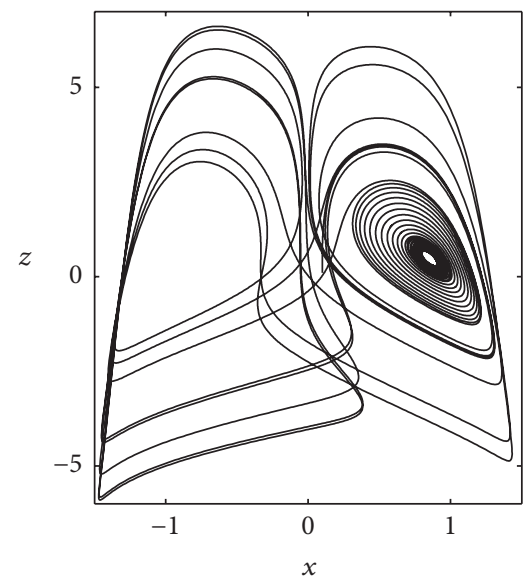

(b)
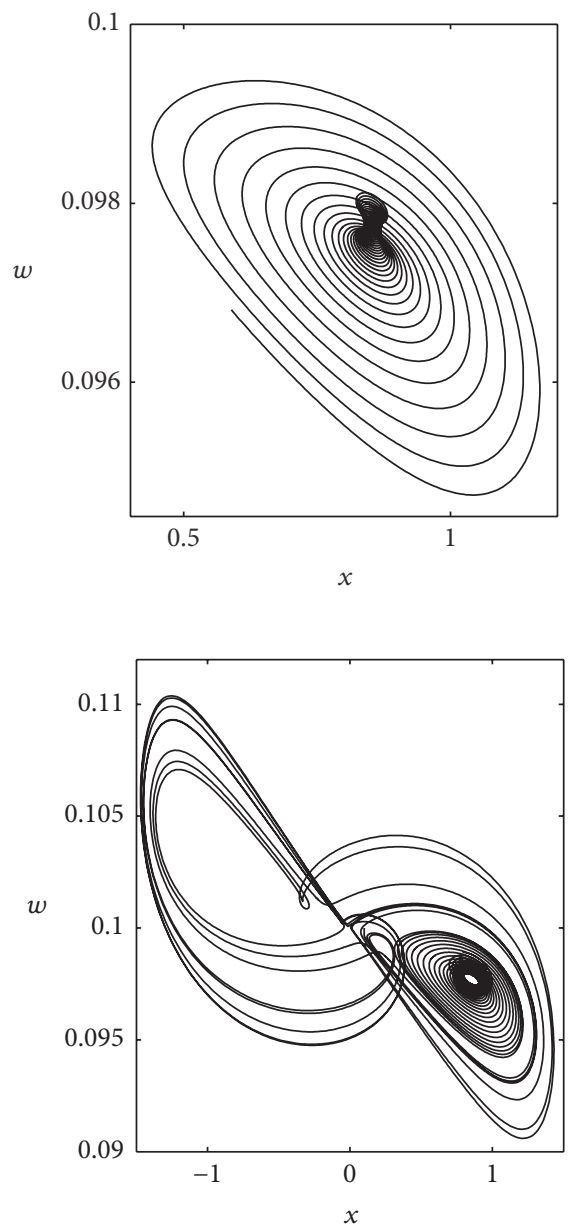

FIGURE 8: The phase portrait in the planes $(x, y),(x, z)$, and $(x, w)$ of fractional-order system (8) at $a=n=1, b=m=0.5, c=0.2$, and $d=2.5$ and for specific values of commensurate fractional-order $q$ : (a) $q=0.945$ and (b) $q=0.948$. The initial conditions used are $(x(0), y(0), z(0), w(0))=(0.1,0.1,0.1,0.1)$. 

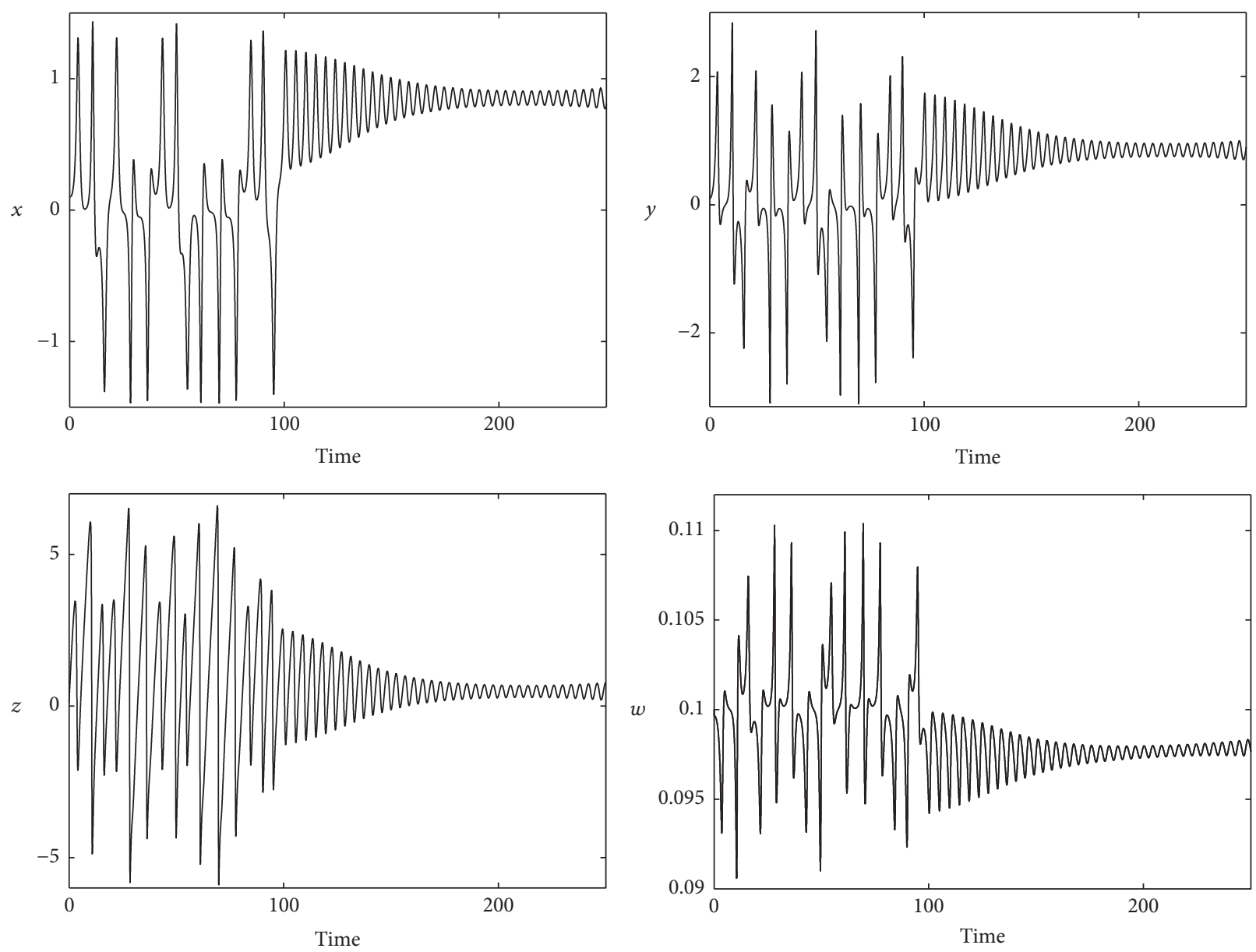

FIGURE 9: The time series of $x(t), y(t), z(t)$, and $w(t)$ for $a=n=1, b=m=0.5, c=0.2, d=2.5$, and $q=0.948$.

Here we can consider the coefficient $k_{x y}$ as an amplitude controller. Attractor of no-equilibrium system (2) is controlled as shown in Figure 7.

\section{Fractional Derivation Effect on the System without Equilibrium}

In this section, we focus on the effect of commensurate fractional derivation on the hyperchaotic system (2) when $a=n=1, b=m=0.5, c=0.2$, and $d=2.5$ (see Figure 1). The fractional-order form of system (2) is obtained by replacing the integer-order derivatives by fractional-order ones. The mathematical description of the fractional-order version of system (2) is expressed as

$$
\begin{aligned}
& \frac{d^{q} x}{d t^{q}}=a(y-x), \\
& \frac{d^{q} y}{d t^{q}}=-b y+n x z+c w,
\end{aligned}
$$

$$
\begin{aligned}
& \frac{d^{q} z}{d t^{q}}=d-e^{x y}, \\
& \frac{d^{q} w}{d t^{q}}=-m y,
\end{aligned}
$$

where $q$ is the derivative order satisfying $0<q<1$. The fractional-order form of system (2) has no-equilibrium points; therefore the effect of fractional derivation on the hyperchaotic system (8) can only be numerically investigated. Here, the Adams-Bashforth-Moulton predictor-corrector scheme is used [41, 42]. This method is based on the Caputo definition of the fractional-order derivative, given by [43]

$$
\frac{d^{q} X_{i}}{d t^{q}}=\frac{1}{\Gamma(q-n)} \int_{0}^{t} \frac{\dot{u}\left(t^{\prime}\right)}{\left(t-t^{\prime}\right)^{q}} d t^{\prime}
$$

where $u=x, y, x, w$ and $\Gamma(\cdot)$ is the Gamma function. We perform the numerical simulations of fractional-order system (8) for different fractional-order $q(0<q<1)$. 

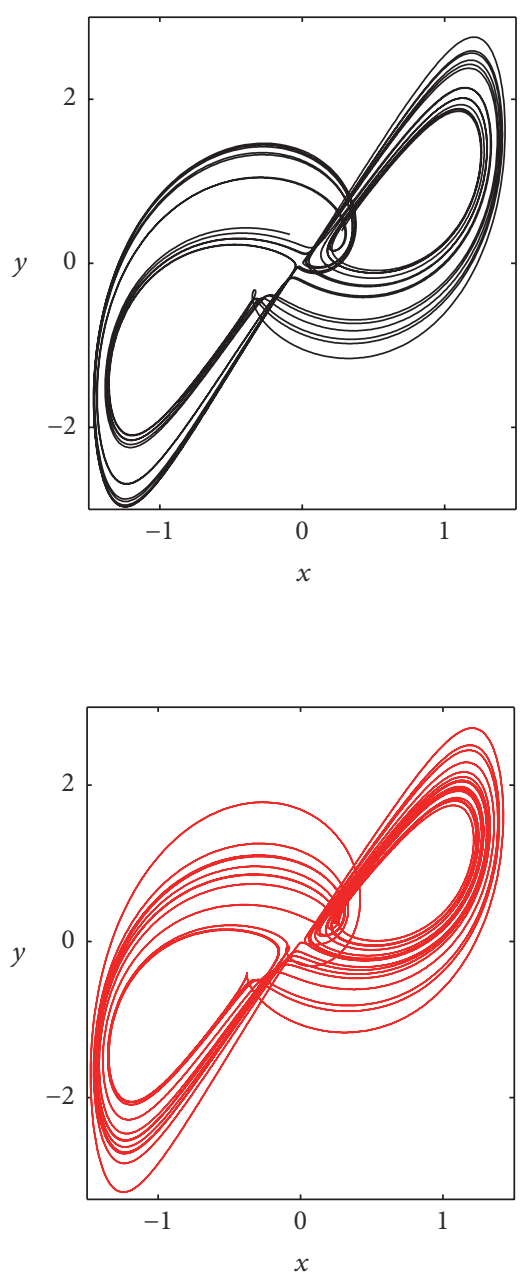

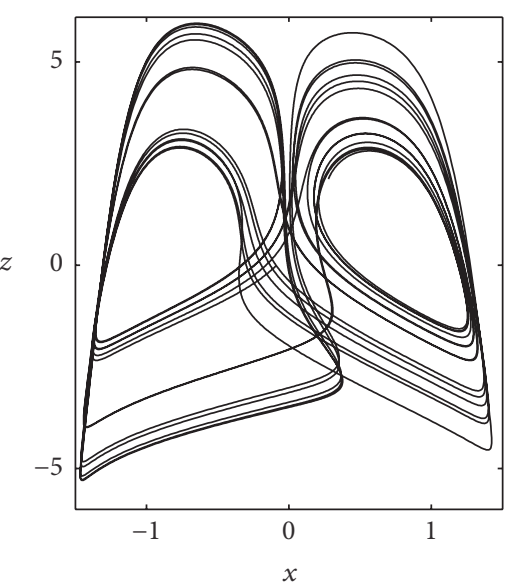

(a)

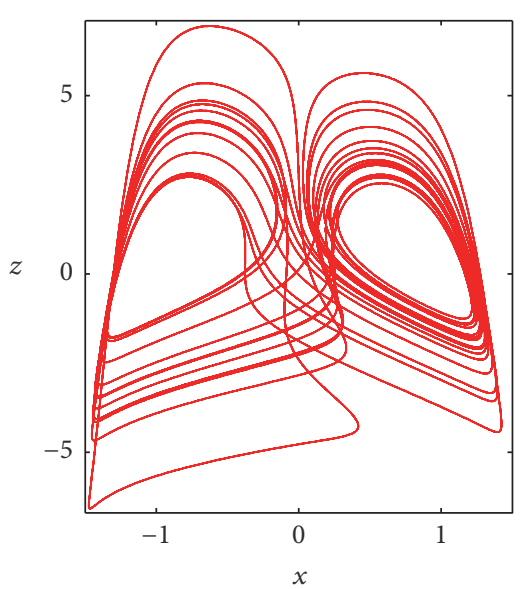

(b)
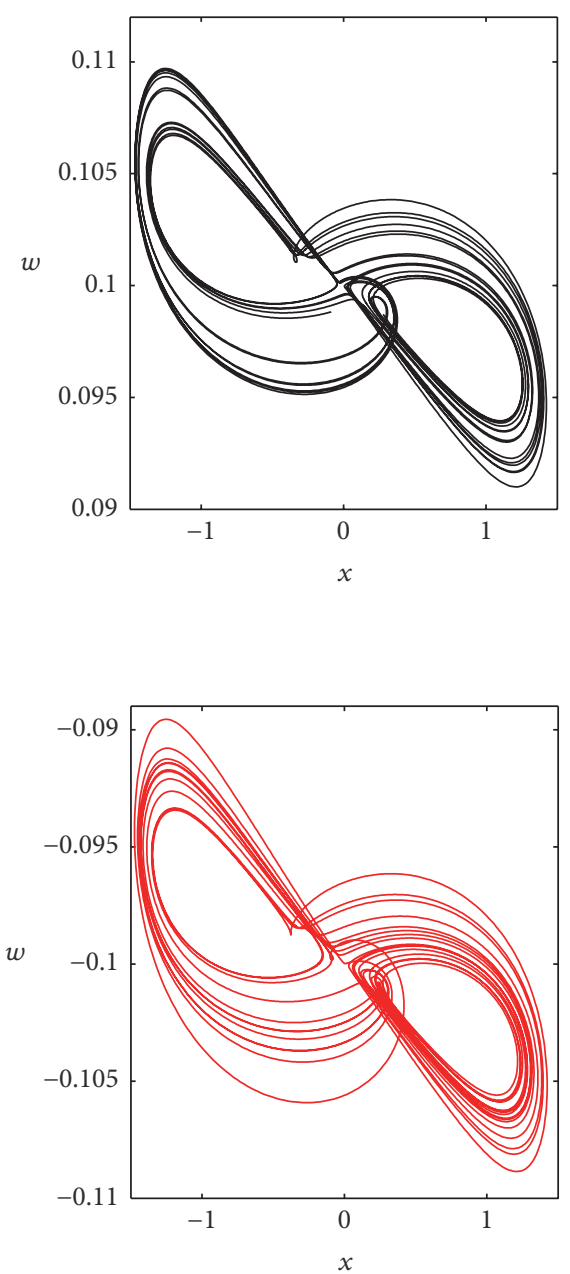

Figure 10: The phase portrait in the planes $(x, y),(x, z)$, and $(x, w)$ of fractional-order system (8) at $a=n=1, b=m=0.5, c=0.2$, $d=2.5$, and $q=0.95$ for specific initial conditions $(x(0), y(0), z(0), w(0))$ : (a) $(0.1,0.1,0.1,0.1)$ (black curves) and (b) $(-0.1,-0.1,0.1,-0.1)$ (red curves).

We present in Figure 8 the phase portraits in the planes $(x, y),(x, z)$, and $(x, w)$ obtained for two specific values of commensurate fractional-order $q$.

For $q=0.945$, the fractional-order system (8) displays a point attractor as shown in Figure 8(a). When the fractional derivative order increases, the fractional-order system (8) presents a transient chaos at $q=0.948$ (see Figure $8(\mathrm{~b})$ ). The transient chaos is confirmed in Figure 9 which depicts the time series of the corresponding phase portraits of fractionalorder system (8) shown in Figure 8(b).

It is clearly seen in Figure 9 that the trajectories of fractional-order system (8) display chaotic behavior for $t<$ 100 . For $t>100$, they converge to an equilibrium point. For $q=0.95$, the phase portraits in the planes $(x, y),(x, z)$, and $(x, w)$ are plotted in Figure 10 .

From Figure 10, one can note that the fractional-order system (8) exhibits bistable double-scroll chaotic attractors at $q=0.95$. The chaotic behavior found in Figure 10 is confirmed in Figure 12 which presents the autocorrelation function of the outputs $x(t), y(t), z(t)$, and $w(t)$ of the corresponding phase portraits of fractional-order system (8) depicted in Figure 10. The coexistence between double-scroll chaotic and quasiperiodic attractors is shown in Figure 11 which presents the phase portraits in the planes $(x, y),(x, z)$, and $(x, w)$ for $q=0.99$.

For $q=0.99$ and using the initial conditions $(x(0)$, $y(0), z(0), w(0))=(0.1,0.1,0.1,0.1)$, double-scroll chaotic attractor is obtained in the fractional-order system (8), while for $q=0.99$ and using the initial conditions $(x(0), y(0)$, $z(0), w(0))=(-0.1,-0.1,0.1,-0.1)$, the fractional-order system (8) exhibits quasiperiodic attractor. The chaotic and quasiperiodic behaviors are confirmed in Figure 12 which presents the autocorrelation function of the outputs $x(t)$, $y(t), z(t)$, and $w(t)$ of the corresponding phase portraits of fractional-order system (8) depicted in Figure 11. In order to know the dynamical behavior found in Figures 10 and 11, we 

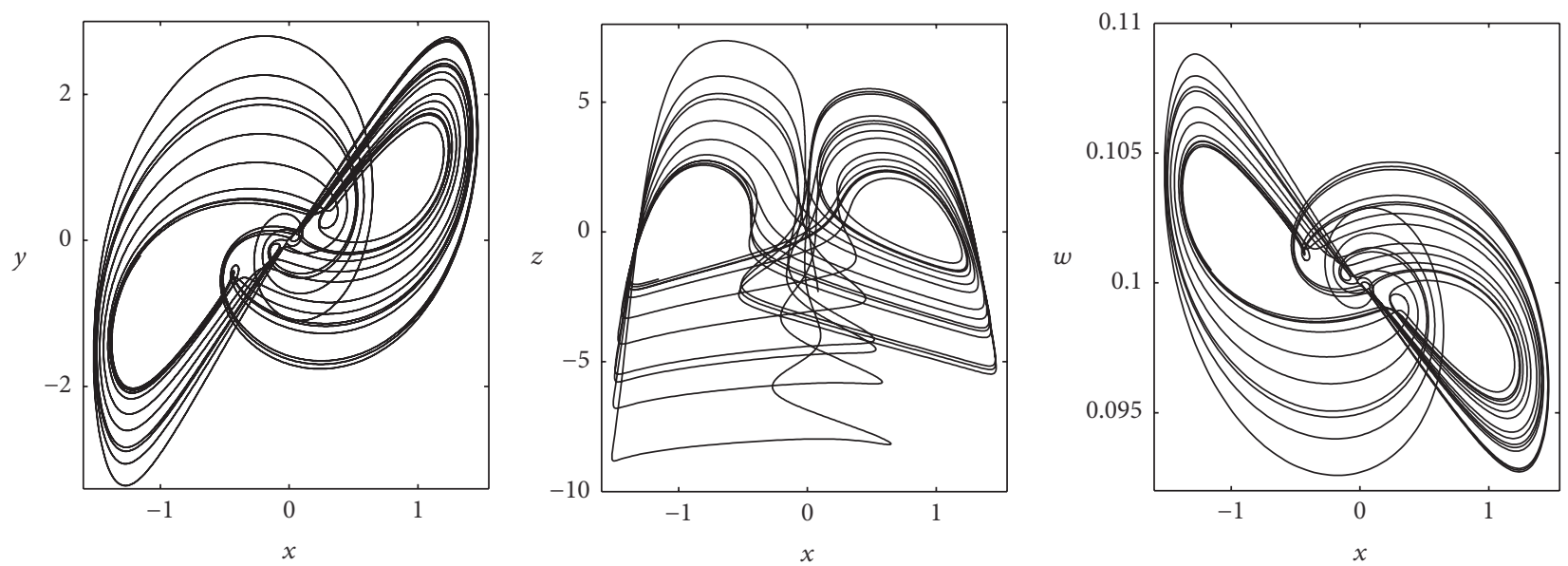

(a)
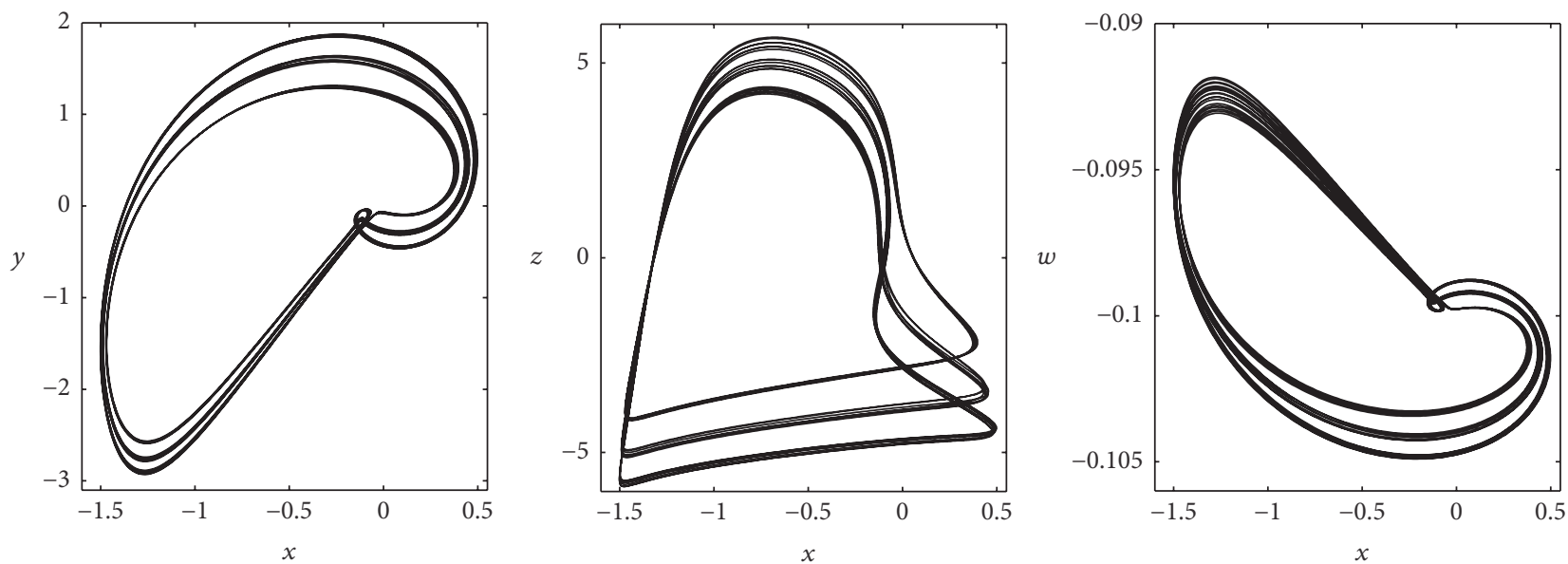

(b)

FIGURE 11: The phase portrait in the planes $(x, y),(x, z)$, and $(x, w)$ of fractional-order system $(8)$ at $a=n=1, b=m=0.5, c=0.2, d=2.5$, and $q=0.99$ for specific initial conditions $(x(0), y(0), z(0), w(0))$ : (a) $(0.1,0.1,0.1,0.1)$ and (b) $(-0.1,-0.1,0.1,-0.1)$.

calculate autocorrelation function of the outputs $x(t), y(t)$, $z(t)$, and $w(t)$. In Figure 12, we present the autocorrelation function

$$
\mathrm{AC}_{u}(t)=\frac{\left\langle[u(t)-\langle u(t)\rangle]\left[u\left(t+t^{\prime}\right)-\langle u(t)\rangle\right]\right\rangle}{[\langle u(t)-\langle u(t)\rangle\rangle]^{2}},
$$

where $u(t)=x(t), y(t), z(t), w(t),\langle u(t)\rangle$ is the mean value of the amplitude along the trajectory, and $t^{\prime}$ is the time shift. The coefficient $\mathrm{AC}_{u}(t)$ is bounded in the range $(-1,1)$ and it stays high for periodic, quasiperiodic, and chaotic cases and decays to zero in the case of the hyperchaotic attractor [44].

\section{Conclusions}

This paper introduces a new system, which has no equilibrium. However, different complex behaviors such as hyperchaos or coexistence of hidden attractors have been observed in such system. In addition, the new system without equilibrium is an amplitude-controllable system which is useful for practical applications. This study has found that commensurate fractional derivation affects the no-equilibrium system. Control and synchronization of such system should be studied in future works.

\section{Conflicts of Interest}

The authors declare that they have no conflicts of interest.

\section{Acknowledgments}

The authors thank Professor GuanRong Chen, Department of Electronic Engineering, City University of Hong Kong, for suggesting many helpful references. 

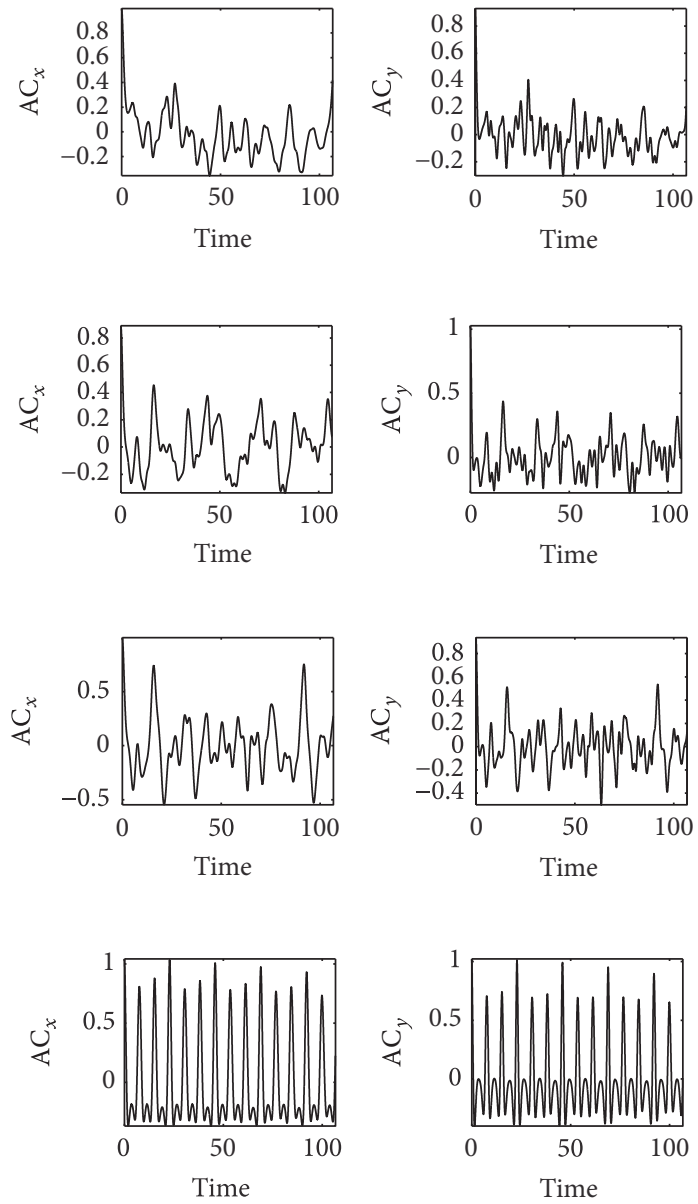

(a1)

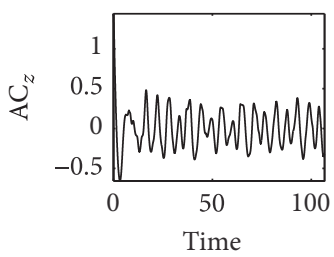

(a2)

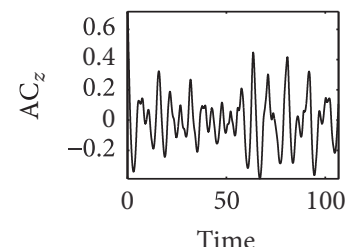

(b1)
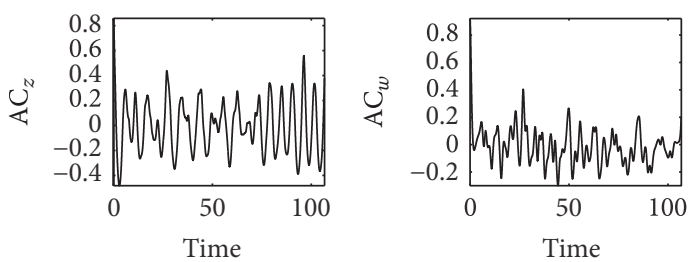

(a1)
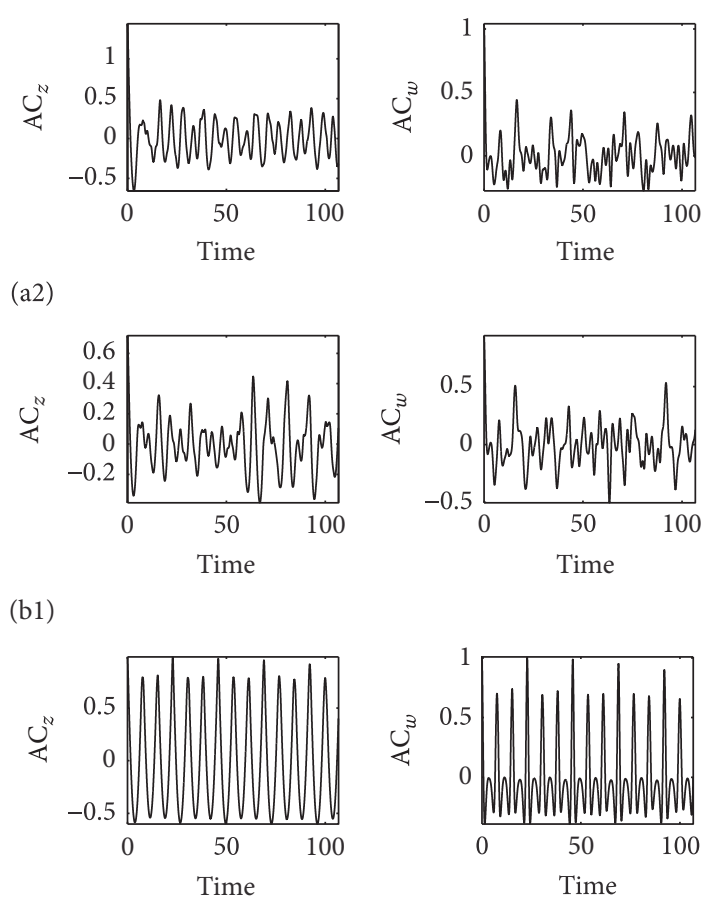

(b2)

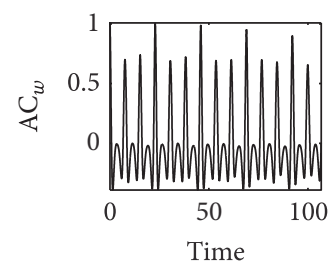

Figure 12: The autocorrelation functions of $x(t), y(t)$, and $z(t)$ at $a=n=1, b=m=0.5, c=0.2$, and $d=2.5$ and for specific values of commensurate-order: (a) $q=0.95$ and (b) $q=0.99$. The curves in (a1) and (b1) are obtained using the initial conditions $(x(0), y(0), z(0), w(0))=(0.1,0.1,0.1,0.1)$ while the curves in (a2) and (b2) are obtained using the initial conditions $(x(0), y(0), z(0), w(0))=$ $(-0.1,-0.1,0.1,-0.1)$.

\section{References}

[1] O. E. Rössler, "An equation for hyperchaos," Physics Letters. A, vol. 71, no. 2-3, pp. 155-157, 1979.

[2] G. Grassi and S. Mascolo, "A system theory approach for designing cryptosystems based on hyperchaos," IEEE Transactions on Circuits and Systems I: Fundamental Theory and Applications, vol. 46, no. 9, pp. 1135-1138, 1999.

[3] Y. Huang and X. Yang, "Hyperchaos and bifurcation in a new class of four-dimensional Hopfield neural networks," Neurocomputing, vol. 69, no. 13-15, pp. 1787-1795, 2006.

[4] V. S. Udaltsov, J. P. Goedgebuer, L. Larger, J. B. Cuenot, P. Levy, and W. T. Rhodes, "Communicating with hyperchaos: the dynamics of a dnlf emitter and recovery of transmitted information," Optics and Spectroscopy, vol. 95, no. 1, pp. 114-118, 2003.

[5] S. Sadoudi, C. Tanougast, M. S. Azzaz, and A. Dandache, "Design and FPGA implementation of a wireless hyperchaotic communication system for secure real-time image transmission," EURASIP Journal on Image and Video Processing, vol. 2013, article 43, pp. 1-18, 2013.
[6] R. Vicente, J. Daudén, P. Colet, and R. Toral, "Analysis and characterization of the hyperchaos generated by a semiconductor laser subject to a delayed feedback loop," IEEE Journal of Quantum Electronics, vol. 41, no. 4, pp. 541-548, 2005.

[7] T. Matsumoto, L. O. Chua, and K. Kobayashi, "Hyperchaos: laboratory experiment and numerical confirmation," Institute of Electrical and Electronics Engineers. Transactions on Circuits and Systems, vol. 33, no. 11, pp. 1143-1149, 1986.

[8] Y. Li, W. K. S. Tang, and G. Chen, "Hyperchaos evolved from the generalized Lorenz equation," International Journal of Circuit Theory and Applications, vol. 33, no. 4, pp. 235-251, 2005.

[9] A. Qi, C. Zhang, and H. Wang, "A switched hyperchaotic system and its FPGA circuitry implementation," Journal of Electronics, vol. 28, no. 3, pp. 383-388, 2011.

[10] S. Dadras, H. R. Momeni, G. Qi, and Z.-1. Wang, "Fourwing hyperchaotic attractor generated from a new $4 \mathrm{D}$ system with one equilibrium and its fractional-order form," Nonlinear Dynamics. An International Journal of Nonlinear Dynamics and Chaos in Engineering Systems, vol. 67, no. 2, pp. 1161-1173, 2012. 
[11] D. Cafagna and G. Grassi, "New 3D-scroll attractors in hyperchaotic Chua's circuits forming a ring," International Journal of Bifurcation and Chaos in Applied Sciences and Engineering, vol. 13, no. 10, pp. 2889-2903, 2003.

[12] A. Chen, J. Lu, J. Lü, and S. Yu, "Generating hyperchaotic Lü attractor via state feedback control," Physica A: Statistical Mechanics and its Applications, vol. 376, pp. 102-108, 2011.

[13] Z. Wang, S. Cang, E. O. Ochola, and Y. Sun, "A hyperchaotic system without equilibrium," Nonlinear Dynamics. An International Journal of Nonlinear Dynamics and Chaos in Engineering Systems, vol. 69, no. 1-2, pp. 531-537, 2012.

[14] V.-T. Pham, F. Rahma, M. Frasca, and L. Fortuna, "Dynamics and synchronization of a novel hyperchaotic system without equilibrium," International Journal of Bifurcation and Chaos in Applied Sciences and Engineering, vol. 24, no. 6, Article ID 1450087, 1450087, 11 pages, 2014.

[15] Z. Wei, R. Wang, and A. Liu, "A new finding of the existence of hidden hyperchaotic attractors with no equilibria," Mathematics and Computers in Simulation, vol. 100, pp. 13-23, 2014.

[16] V.-T. Pham, C. Volos, and L. V. Gambuzza, "A memristive hyperchaotic system without equilibrium," Scientific World Journal, vol. 2014, Article ID 368986, 2014.

[17] V. T. Pham, S. Vaidyanathan, C. K. Volos, and S. Jafari, "Hidden attractors in a chaotic system with an exponential nonlinear term," European Physical Journal: Special Topics, vol. 224, no. 8, Article ID A1507, pp. 1507-1517, 2015.

[18] Z. Wang, J. Ma, S. Cang, Z. Wang, and Z. Chen, "Simplified hyper-chaotic systems generating multi-wing non-equilibrium attractors," Optik, vol. 127, no. 5, pp. 2424-2431, 2016.

[19] B. C. Bao, H. Bao, N. Wang, M. Chen, and Q. Xu, "Hidden extreme multistability in memristive hyperchaotic system," Chaos, Solitons Fractals, vol. 94, pp. 102-111, 2017.

[20] D. Cafagna and G. Grassi, "Fractional-order systems without equilibria: the first example of hyperchaos and its application to synchronization," Chinese Physics B, vol. 24, no. 8, Article ID 080502, 2015.

[21] K. Rajagopal, A. Karthikeyan, and A. K. Srinivasan, "FPGA implementation of novel fractional-order chaotic systems with two equilibriums and no equilibrium and its adaptive sliding mode synchronization," Nonlinear Dynamics. An International Journal of Nonlinear Dynamics and Chaos in Engineering Systems, vol. 87, no. 4, pp. 2281-2304, 2017.

[22] G. A. Leonov and N. V. Kuznetsov, "Hidden attractors in dynamical systems. From hidden oscillations in HilbertKolmogorov, Aizerman, and KALman problems to hidden chaotic attractor in Chua circuits," International Journal of Bifurcation and Chaos in Applied Sciences and Engineering, vol. 23, no. 1, Article ID 1330002, 1330002, 69 pages, 2013.

[23] D. Dudkowski, S. Jafari, T. Kapitaniak, N. V. Kuznetsov, G. A. Leonov, and A. Prasad, "Hidden attractors in dynamical systems," Physics Reports. A Review Section of Physics Letters, vol. 637, pp. 1-50, 2016.

[24] X. Wang and G. Chen, "Constructing a chaotic system with any number of equilibria," Nonlinear Dynamics. An International Journal of Nonlinear Dynamics and Chaos in Engineering Systems, vol. 71, no. 3, pp. 429-436, 2013.

[25] S. Brezetskyi, D. Dudkowski, and T. Kapitaniak, "Rare and hidden attractors in Van der Pol-Duffing oscillators," European Physical Journal: Special Topics, vol. 224, no. 8, pp. 1459-1467, 2015.

[26] P. R. Sharma, M. D. Shrimali, A. Prasad, N. V. Kuznetsov, and G. A. Leonov, "Control of multistability in hidden attractors," Euro- pean Physical Journal: Special Topics, vol. 224, no. 8, pp. 14851491, 2015.

[27] Z. T. Zhusubaliyev and E. Mosekilde, "Multistability and hidden attractors in a multilevel DC/DC converter," Mathematics and Computers in Simulation, vol. 109, pp. 32-45, 2015.

[28] Z. Wei and Q. Yang, "Dynamical analysis of a new autonomous 3-D chaotic system only with stable equilibria," Nonlinear Analysis. Real World Applications. An International Multidisciplinary Journal, vol. 12, no. 1, pp. 106-118, 2011.

[29] N. Levinson, "Transformation theory of non-linear differential equations of the second order," Annals of Mathematics. Second Series, vol. 45, pp. 723-737, 1944.

[30] G. A. Leonov, N. V. Kuznetsov, and T. N. Mokaev, "Homoclinic orbits, and self-excited and hidden attractors in a Lorenz-like system describing convective fluid motion," European Physical Journal: Special Topics, vol. 224, no. 8, pp. 1421-1458, 2015.

[31] A. Wolf, J. B. Swift, H. L. Swinney, and J. A. Vastano, "Determining Lyapunov exponents from a time series," Physica D. Nonlinear Phenomena, vol. 16, no. 3, pp. 285-317, 1985.

[32] P. Frederickson, J. L. Kaplan, E. D. Yorke, and J. A. Yorke, "The Liapunov dimension of strange attractors," Journal of Differential Equations, vol. 49, no. 2, pp. 185-207, 1983.

[33] N. V. Kuznetsov, T. A. Alexeeva, and G. A. Leonov, "Invariance of LYApunov exponents and Lyapunov dimension for regular and irregular linearizations," Nonlinear Dynamics. An International Journal of Nonlinear Dynamics and Chaos in Engineering Systems, vol. 85, no. 1, pp. 195-201, 2016.

[34] N. V. Kuznetsov, "The Lyapunov dimension and its estimation via the LEOnov method," Physics Letters. A, vol. 380, no. 25-26, pp. 2142-2149, 2016.

[35] G. A. Leonov, N. V. Kuznetsov, N. A. Korzhemanova, and D. V. Kusakin, "Lyapunov dimension formula for the global attractor of the Lorenz system," Communications in Nonlinear Science and Numerical Simulation, vol. 41, pp. 84-103, 2016.

[36] A. Buscarino, L. Fortuna, M. Frasca, and L. V. Gambuzza, "A chaotic circuit based on Hewlett-Packard memristor," Chaos. An Interdisciplinary Journal of Nonlinear Science, vol. 22, no. 2, Article ID 023136, 023136, 9 pages, 2012.

[37] C. Li, J. C. Sprott, Z. Yuan, and H. Li, "Constructing chaotic systems with total amplitude control," International Journal of Bifurcation and Chaos in Applied Sciences and Engineering, vol. 25, no. 10, Article ID 1530025, 1530025, 14 pages, 2015.

[38] C. Li and J. C. Sprott, "Variable-boostable chaotic flows," Optik, vol. 127, no. 22, pp. 10389-10398, 2016.

[39] C. Li and J. C. Sprott, "Amplitude control approach for chaotic signals," Nonlinear Dynamics. An International Journal of Nonlinear Dynamics and Chaos in Engineering Systems, vol. 73, no. 3, pp. 1335-1341, 2013.

[40] C. Li and J. C. Sprott, "Finding coexisting attractors using amplitude control," Nonlinear Dynamics. An International Journal of Nonlinear Dynamics and Chaos in Engineering Systems, vol. 78, no. 3, pp. 2059-2064, 2014.

[41] W. Deng, "Short memory principle and a predictor-corrector approach for fractional differential equations," Journal of Computational and Applied Mathematics, vol. 206, no. 1, pp. 174-188, 2007.

[42] K. Diethelm, N. J. Ford, and A. D. Freed, "A predictor-corrector approach for the numerical solution of fractional differential equations," Nonlinear Dynamics. An International Journal of Nonlinear Dynamics and Chaos in Engineering Systems, vol. 29, no. 1-4, pp. 3-22, 2002. 
[43] R. Caponetto, G. Dongola, L. Fortuna, and I. Petras, "Fractional Order Systems: Modeling and Control Applicationss, World Scientific," 2010, Singapore.

[44] P. Perlikowski, S. Yanchuk, M. Wolfrum, A. Stefanski, P. Mosiolek, and T. Kapitaniak, "Routes to complex dynamics in a ring of unidirectionally coupled systems," Chaos. An Interdisciplinary Journal of Nonlinear Science, vol. 20, no. 1, 013111, 10 pages, 2010. 


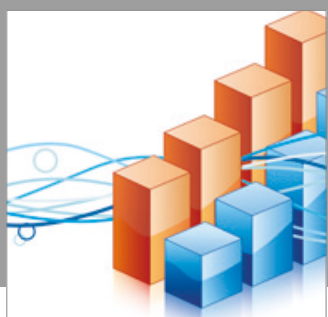

Advances in

Operations Research

vatersals

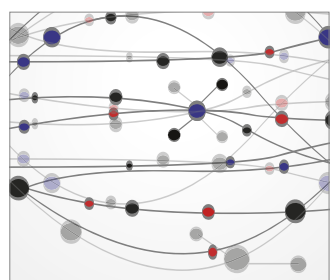

\section{The Scientific} World Journal
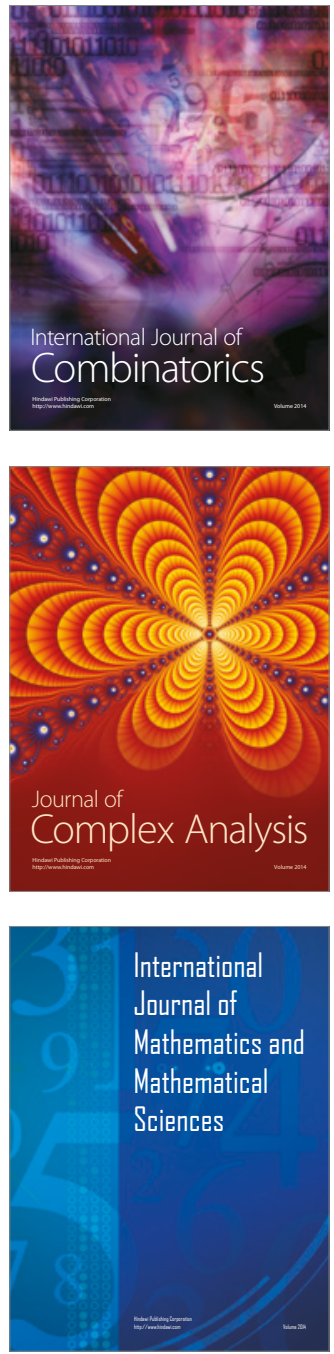
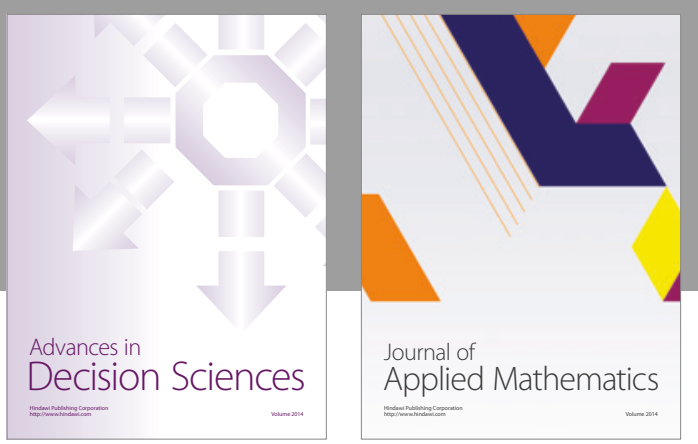

Algebra

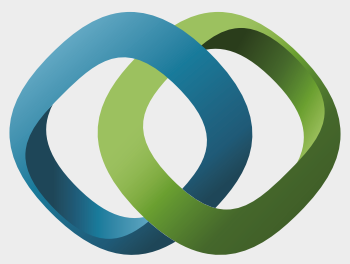

\section{Hindawi}

Submit your manuscripts at

https://www.hindawi.com
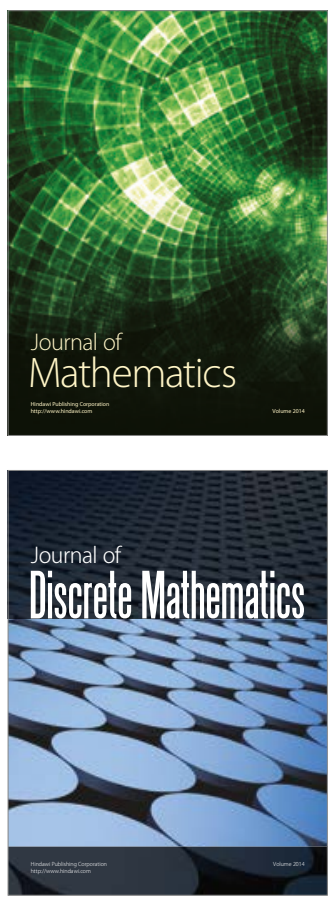

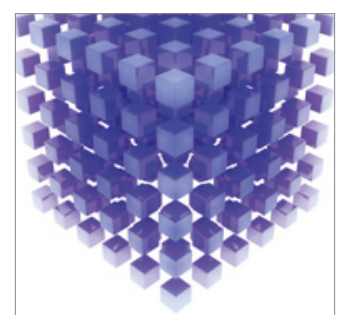

Mathematical Problems in Engineering
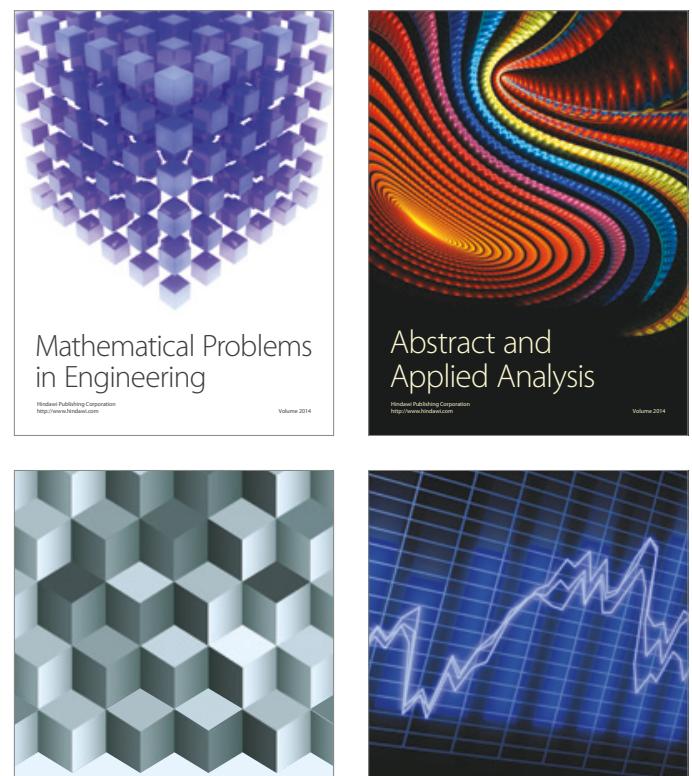

Journal of

Function Spaces

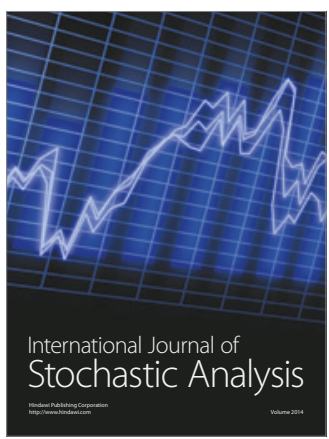

Probability and Statistics
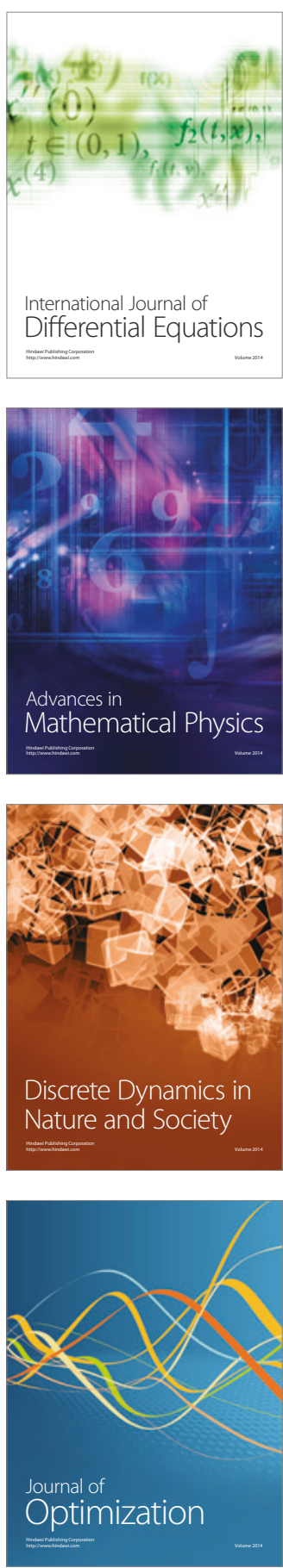